\title{
Labyrinthe
}

19 | 2004 (3)

Le Bel Aujourd'hui

\section{Les faces cachées du texte scientifique}

Nathalie Vuillemin

\section{(2) OpenEdition}

Journals

Édition électronique

URL : http://journals.openedition.org/labyrinthe/249

DOI : $10.4000 /$ labyrinthe. 249

ISSN : 1950-6031

Éditeur

Hermann

Édition imprimée

Date de publication : 15 décembre 2004

Pagination : 131-134

Référence électronique

Nathalie Vuillemin, «Les faces cachées du texte scientifique », Labyrinthe [En ligne], 19 | 2004 (3), mis en ligne le 19 juin 2008, consulté le 03 mai 2019. URL : http://journals.openedition.org/labyrinthe/249 ; DOI : 10.4000/labyrinthe.249

Propriété intellectuelle 


\title{
LES FACES CACHÉES DU TEXTE SCIENTIFIQUE*
}

\author{
Nathalie VuILLEMIN \\ nathalie.vuillemin@unine.ch
}

Entreprendre une thèse de littérature française sur un corpus de textes savants consacrés à des thèmes a priori aussi peu alléchants que l'histoire naturelle des minéraux, des vers, des insectes ou des coquillages peut sembler quelque peu paradoxal; prétentieuse, l'ambition d'apporter, grâce à ces écrits, de nouvelles perspectives critiques sur la littérature des Lumières. C'est pourtant le projet que je poursuis, dans une recherche intitulée «Zoophytes, polypes, fossiles... L'écriture de l'inconnu scientifique entre placere et docere dans la seconde moitié du XVIII ${ }^{e}$ siècle (1740-1805)». Il s'agit d'examiner les fonctionnements de l'écriture savante, en portant une attention toute particulière aux dimensions esthétiques qui parasitent l'entreprise cognitive, l'enjolivent, la détournent de ses visées premières ou, à l'inverse, s'en font le vecteur essentiel. L'Histoire naturelle de Buffon surgit évidemment comme l'exemple le plus flagrant - et le mieux connu - des rencontres entre placere et docere dans le texte «scientifique» de l'époque. Mais j'ai tenté, pour donner des problèmes une vision qui dépasse les spécificités d'un grand nom, de m'attaquer à un vaste ensemble de représentations du savoir, des mémoires de l'Académie des sciences, à la vision très poétique que Bernardin de Saint-Pierre produit dans ses Études de la nature, en passant par des traités spécialisés, comme celui d'Abraham Trembley sur le polype', ou des ouvrages de vulgarisation.

Plusieurs ouvrages critiques, ces dernières années, ont souligné l'importance des stratégies «littéraires » mises en place par les savants

\footnotetext{
* Cette thèse est inscrite à l'université de Neuchâtel (Suisse) en littérature française du XVIII ${ }^{\mathrm{e}}$ siècle. Elle est dirigée par le professeur Claire Jaquier. Une co-direction avec le professeur Jean-Paul Sermain (Paris III) est prévue. La soutenance aura lieu fin 2005.

1. Mémoires pour servir à l'histoire d'un genre de polypes d'eau douce, à bras en forme de cornes, Leyde, Verbeek, 1744.
} 
de l'Ancien Régime dans les récits de découvertes, d'expériences ou d'observations qu'ils livrent à leurs confrères, à leurs élèves ou au grand public ${ }^{2}$. Les travaux d'historiens comme Michel Foucault ou Jean Starobinski ont par ailleurs rendu attentifs aux échanges permanents qu'entretiennent, au XVIII ${ }^{\mathrm{e}}$ siècle, les idées savantes et «littéraires », échanges que Claude Reichler, dans une récente conférence, représentait sous la forme de «nœuds» dans lesquels s'enchaînent et s'inspirent mutuellement les différents imaginaires de la nature ${ }^{3}$.

C'est également de ces nœuds dont il est question ici. Parallèlement à un regard rationnel et analytique, à la volonté de mettre à nu les mécanismes naturels dans un texte idéalement transparent, le savant des Lumières développe une perspective esthétisante sur des êtres dont l'extraordinaire ne frappe souvent que lui. Cette vision se modèle alors dans une rhétorique sensible et subjective, qui s'oppose par définition à l'objectivité que s'assigne le programme scientifique, mais rejoint, en revanche, la réflexion des poéticiens, grammairiens et logiciens de l'époque sur les devenirs de l'éloquence. Se posent alors un certain nombre de questions : cette dimension esthétique, ou poétique, relèvet-elle de simples stratégies discursives? Traduit-elle un attachement profond à la tradition contemplative, telle qu'elle se manifeste dans des ouvrages comme ceux de l'Abbé Pluche ou, plus avant dans le siècle, de Charles Bonnet ${ }^{4}$ ? Est-elle au contraire finalisée, dans une sorte de refus d'une vision purement rationnelle de la nature? Apparaît-elle privilégiée dans certains genres d'ouvrages?

2. Depuis le début des années 1980, les chercheurs anglo-saxons, notamment, ont multiplié les études de ce type. Voir par exemple Ludmilla Jordanova (ed.), Languages of Nature. Critical Essays on Science and Literature, London, Free Association Books, 1986 ; Peter Dear (ed.), The Literary Structure of Scientific Argument. Historical Studies, Philadelphia, University of Pennsylvania Press, 1991. En France, on mentionnera en particulier les études suivantes: Fernand Hallyn, La Structure poétique du monde : Copernic, Kepler, Paris, Seuil, 1987; Les Structures rhétoriques de la science, Paris, Seuil, 2004. Christian Licoppe, La Formation de la pratique scientifique. Le discours de l'expérience en France et en Angleterre (1630-1820), Paris, La Découverte, 1996.

3. Claude Reichler, «L'air et les météores au tournant du siècle. Une fascination multiple (sciences, voyages, peinture, littérature) », conférence présentée le samedi 15 mai 2004 à l'université de Paris IIISorbonne nouvelle, dans le cadre des travaux du Cerlav 18.

4. Antoine Pluche, Le Spectacle de la nature, ou entretiens sur les particularités de l' histoire naturelle, Paris, 1732-1746; Charles Bonnet, Contemplation de la nature, Amsterdam, 1764. 
L'examen d'un corpus important de textes savants ${ }^{5}$ des années 1740 à 1805 m'a conduite à approfondir ces questions par le biais d'une analyse métadiscursive: les savants, dans leurs déclarations préliminaires, avertissements, prospectus, parfois même au détour d'un quelconque mémoire ou article de dictionnaire, débattent en effet vivement de la forme que doit prendre l'interrogation de la nature. Un premier aspect de mon travail consiste donc à définir le texte scientifique idéal, tel que l'imaginent les auteurs de l'époque; les recherches contemporaines en matière d'esthétique et de poétique sont ici des références essentielles, qui mettent en relief les spécificités du discours savant, mais également la manière dont on reçoit les théories d'autres domaines, s'en inspire ou les rejette. Dans un second temps, l'analyse de l'écrit lui-même, de ses articulations, des images ou des analogies qu'on y développe pour expliquer un phénomène, conduit à confronter le modèle théorique aux réalisations concrètes. C'est dans cette seconde phase que surgissent les décalages rhétoriques que je mentionnais plus haut. Il s'agit alors de découvrir leur fonction, et les enjeux philosophiques qu'ils révèlent - ou qu'ils cachent. Le savant s'assigne, certes, la tâche de dévoiler à ses contemporains une nature conçue comme un ensemble de mécanismes qui, à défaut d'être toujours compréhensibles à l'homme, s'avèrent au moins sujets à être décrits. La transparence idéale vers laquelle tend ce tableau est-elle possible? Que suppose-t-elle quant au regard ultérieur sur l'univers? Comment donner à comprendre, à voir, et à admirer dans un même mouvement ? Autant d'interrogations qu'il convient, avant d'y répondre, de lire dans les textes, pour aboutir peu à peu à une compréhension des représentations scientifiques de la nature au XVIII ${ }^{\mathrm{e}}$ siècle.

Une telle recherche exploite conjointement les méthodes de l'analyse discursive, de l'histoire des idées et de l'histoire des sciences. Si le constat semble évident, ses implications n'apparaissent toutefois clairement que lorsque les premiers obstacles méthodologiques viennent entraver la mise en forme de l'analyse. En amont des choix textuels des auteurs, on trouve bien entendu un contexte propre au microcosme scientifique: le savoir, mais surtout les méthodes d'une

5. Par «textes savants», j'entends dans cette recherche des textes dont les thématiques sont issues des observations et des questionnements scientifiques de l'époque. 
époque, les règles tacites de la communauté savante, la définition du public, tous « [c]es facteurs qui, à un moment donné de l'histoire, interviennent dans le 'choix rationnel' opéré par le savant ${ }^{6} »$, apparaissent comme autant de codes qu'il convient de déchiffrer. L'historien des sciences attend la description circonstanciée de ces « raisons » du texte; ce dernier élément ne l'intéresse que dans la mesure où il témoigne - ou résulte - de pratiques concrètes qui constituent, à proprement parler, l'histoire de la science.

L'histoire des idées telle qu'on l'envisage avec Jacques Roger ou Michel Delon souhaite au contraire étudier l'ouvrage savant non seulement comme documentation d'une époque, mais avant tout comme écriture, répondant à certaines règles qui modèlent la pensée. La logique du texte révèle alors des significations qui dépassent largement les conditions de sa production ${ }^{7}$. C'est évidemment selon cette optique que s'oriente ma recherche; mais force est de le constater, les éléments «contextuels» que met à jour l'histoire des sciences doivent être intégrés à l'analyse, rappelés au lecteur, résumés en évitant de les minimiser. Si tout chercheur s'interroge perpétuellement sur les destinataires de ses travaux, la question devient ici la raison même du travail : par nature, mon corpus appelle d'abord (les critiques de) l'historien des sciences; l'analyse, elle, s'adresse au littéraire, au linguiste, à l'historien des idées. Utiliser à bon escient un type de savoir au service d'un autre, tel est le défi qu'il s'agit de relever, et de transmettre.

Idéalement, cette thèse se veut ainsi un «lieu d'échanges et de confrontations ${ }^{8} »$ entre des univers qui, dans l'état actuel de la recherche universitaire, se perçoivent encore trop souvent comme étrangers, voire antagonistes l'un à l'autre. Tout ardu qu'il puisse d'abord paraître, le texte scientifique est un aspect de la littérature des Lumières qui, peu exploré, invite à de nombreuses enquêtes, et enrichit indubitablement la compréhension des grands débats philosophiques et poétiques de l'époque.

6. Jacques Roger, «L'histoire des sciences: problèmes et pratiques. Histoire des sciences, histoire des mentalités, micro-histoire", dans Les Sciences de la vie dans la pensée française au XVIII siècle, Paris, Albin Michel, éd. 1993, p. XIX-XXXIX ; p. XXIX pour cette citation.

7. Michel Delon, L'Idée d'énergie au tournant des Lumières (1770-1820), Paris, Puf, 1988, p. 16.

8. Ibid., p. 15 . 